\title{
Governance in urban agriculture: challenges and opportunities
}

\author{
Isabel de Felipe ${ }^{1}$, Teresa Briz ${ }^{2}$
}

${ }^{1,2}$ Universidad Politécnica de Madrid (UPM) - Avda. Complutense, s/n. 28040, Madrid - Espanha.

Email: Isabel.defelipe@upm.es

Received: February $25^{\text {th }}, 2018$.

\begin{abstract}
Urban agriculture is a traditional activity that is getting new support in many cities. However, is facing difficulties resulting from inadequate governance. We identified eight instruments used to achieve a sustainable urban agriculture, such as food security, economic development, environment, health, relationships, regulatory, social and others. Each city requires specific governance, looking for synergies. The paper gives a general description of international cases.
\end{abstract}

Accepted: March $19^{\text {th }}, 2018$.

Copyright (C)2016 by authors and Institute of Technology Galileo of Amazon (ITEGAM).

This work is licensed under the Creative Commons Attribution International

License (CC BY 4.0).

http://creativecommons.org/licenses/by/4.0/

Keywords: Urban agriculture, governance, synergies, efficiency.

\section{La gobernanza en la agricultura urbana: retos y oportunidades}

\section{RESUMEN}

La Agricultura Urbana es una actividad tradicional que está tomando un fuerte impulso en muchas metrópolis. No obstante, se enfrenta a dificultades derivadas de una gobernanza inadecuada. Identificamos los ocho instrumentos utilizados para lograr una agricultura urbana sostenible, integrados en una estrella multifocal que incluye la seguridad alimentaria, el desarrollo económico, medio ambiente, salud, relaciones sociales, normativas y otros. Cada ciudad requiere una gobernanza específica, lo que exige integrar a todas las fuerzas vivas de su entorno, buscando las sinergias y soslayando los puntos de conflicto. Se hace una descripción de casos a nivel internacional, unas reflexiones finales y unas propuestas.

Palabras clave: Agricultura urbana, gobernanza, sinergias, eficiencia.

\section{INTRODUCCIÓN}

Informes de organizaciones internacionales, como FAO, sobre el reto de alimentar a la Humanidad en las próximas décadas, muestran que en 2050 la población mundial alcanzará los nueve mil millones de personas, lo que implicará incrementar la producción de alimentos un $70 \%$. Incluso hoy día, el hambre en el mundo es la gran asignatura pendiente en nuestra agenda.

Por ello, la tarea de producción de alimentos concierne a todos, y no se puede excluir ninguna fuente,de esa gran labor. La agricultura rural tradicional seguirá teniendo la responsabilidad estratégica del abastecimiento alimentario, sin embargo, hay sectores y actividades donde pueden reorganizarse las funciones entre agricultura rural y agricultura urbana (AU).

El nuevo marco en el que se desarrollan las actividades agrarias no contempla solamente la oferta alimentaria, sino que se incluye el cambio climático, las huellas de carbono, energética e hídrica, el proceso acelerado de urbanizaciones y las nuevas tecnologías en diseño y construcción que permiten una agricultura urbana en altura de forma intensiva.
La idea de agricultura en altura fue expuesta en 1999 por Dickson Despommier, de la Universidad de Columbia de Nueva York [1] y describió sus beneficios. La agricultura urbana de altura (AUA) cuenta con nuevos planteamientos e innovaciones que le permiten ocupar espacios desaprovechados.

Los cultivos hidropónicos combinados con la piscicultura (agropiscicultura), la utilización de gases residuales de centrales de calefacción, el empleo de Light Emitting Diodes (LED), placas solares y otros medios, permiten mejorar los rendimientos y disminuir costes al utilizar menos agroquímicos y herbicidas.

La agricultura de proximidad, ocupación de ocio, integración social y la ubicación en el "kilómetro cero", reduciendo costes de transporte, son algunas de sus cualidades.

No obstante, hay también fuertes críticas a la AUA ante los efectos contaminantes de metales pesados en los productos urbanos, la escasa capacidad de aplicar economías de escala y la limitación de ciertas actividades agrícolas (arboricultura, cereales) que requieren mayor sustrato o extensiones de cultivo, así como ciertos sectores ganaderos (vacuno y porcino) con obstáculos difíciles de superar. 
No se trata, por lo tanto, de plantear la alternativa de la agricultura urbana versus la rural, sino como complementarias, seleccionando aquellos cultivos más idóneos.

Existe para algunos la idea de soberanía alimentaria para las grandes metrópolis, pero consideramos que, hoy en día, es una fantasía. Sin embargo, hemos de reconocer que en los últimos años hay una etapa de despegue, con proyectos emblemáticos que marcan unas nuevas perspectivas. Los casos de Plantagón (Suecia) o SkyGreens (Singapur) son ejemplos paradigmáticos. En conclusión, todas las agriculturas, tanto urbana como rural, pueden y deben colaborar en la estratégica tarea de abastecernos de alimentos.

\section{LA GOBERNANZA EN LA AGRICULTURA URBANA}

La idea general sobre la construcción de un simulador de procesos que considere a la operación unitaria de evaporación, se basa en la disposición de equipos piloto de uno y doble efecto para la realización de prácticas experimentales con alto costo en materia prima y consumo de energía, En este sentido, el desarrollo de un laboratorio virtual permitira la reducción de costos de operación, y la posibilidad de realizar prácticas virtuales y la simulación de la operación en tiempo real para evaluar el desempeño del equipo ante diferentes condiciones de operación.

Hemos de conseguir instituciones que ayuden a resolver los problemas ciudadanos mediante su participación y corresponsabilidad directa, con la mayor eficiencia y a todos los niveles. Para ello utilizamos el término de"gobernanza", que tiene numerosas acepciones. Entre ellas podemos señalar: "Arte o manera de gobernar que se propone como objetivo el logro de un desarrollo económico, social e institucional duradero, promoviendo un sano equilibrio entre el Estado, la sociedad civil y el mercado de la economía" [2]

Entre los aspectos estratégicos de la gobernanza socioeconómica (UNPAN, 2014), se pueden señalar la capacidad para gestionar el dialogo entre los ciudadanos en las políticas de cambio, explorando los mecanismos que llevan a una participación eficiente y directa, con seguimiento y auditoria de los programas.

Simultáneamente, la gobernanza debe afrontar los problemas derivados de la división de competencias administrativas [3], hecho que suele ser habitual en las Administraciones Locales, interaccionando con las Regionales y Nacionales, lo que supone una barrera adicional en la gestión de áreas verdes urbanas.

La gobernanza contempla, asimismo, el análisis de la mayor participación de actores no gubernamentales, que entran en conflicto de intereses, lo que anula o demora la aplicación de políticas sociales y medioambientales [4]. El fenómeno es habitual en el diseño de los planes urbanísticos, que deben compaginar intereses diversos y contrapuestos, con cuotas de poder muy heterogéneas. La posición de la $\mathrm{AU}$ parte con desventaja en los momentos actuales, aunque las circunstancias pueden cambiar en el próximo futuro, con el aumento del poder demográfico y político de las ciudades.

Hay argumentos que deben transformarse en normas. Hasta ahora, los movimientos verdes urbanos se basaban más en la emoción que en la información, pero los estudios socioeconómicos muestran las necesidades alimentarias, problemas de contaminación, medio ambiente y salud, aumento de residuos y otros aspectos que hacen que el modelo urbano actual sea insostenible y donde una gobernanza eficiente puede ayudar a reenfocar la situación vigente. Tema de interés es la evaluación y comparación de la resiliencia de las distintas comunidades urbanas para adaptarse a las nuevas circunstancias de explosión demográfica, dependencia exterior y sostenibilidad del sistema. Pueden considerarse distintos tipos de resiliencia, desde la medioambiental, a la tecnológica, negocio-emprendedora o jurídica, entre otras [2].

La lucha contra el hambre en barrios marginados, mejorando su dieta alimentaria, debe combinarse con la educación y la formación, la vertebración social, el empoderamiento y la cuestión de género, el desarrollo socioeconómico, la creación de empleo, las áreas de recreo y el ejercicio físico, el medio ambiente y la biodiversidad.

La estrella de la gobernanza requiere optimizar la coordinación de políticas y actividades en las siguientes áreas.

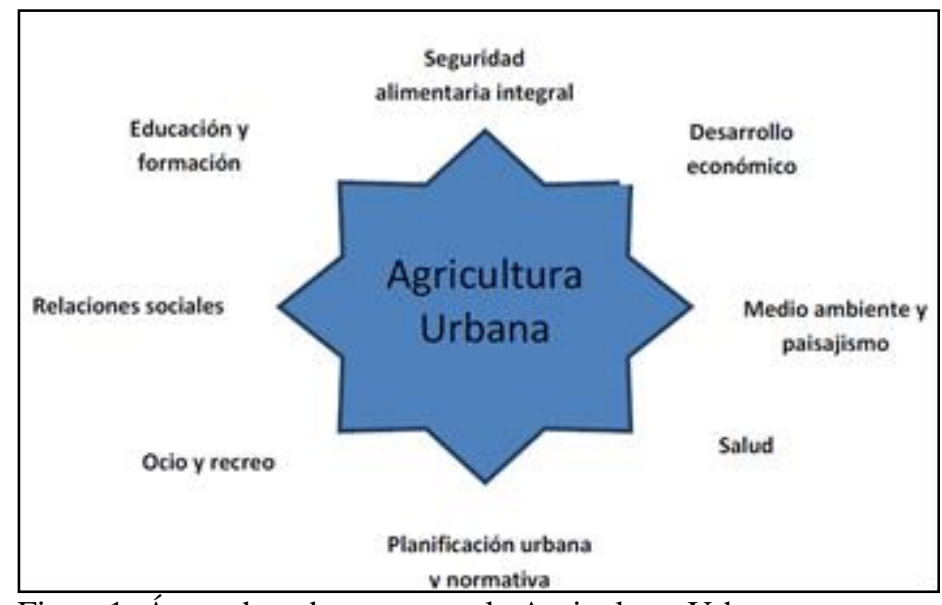

Figura1: Áreas de gobernanza en la Agricultura Urbana.

Fuente: Los autores, (2018).

\section{a) Seguridad alimentaria integral.}

Incluye el abastecimiento de alimentos y su inocuidad. Habitualmente la gestión corresponde a los Ministerios de Agricultura y Sanidad y a los departamentos correspondientes de la Administración local. El planteamiento de la gobernanza en este ámbito, varía significativamente según el grado de desarrollo de los países. En Berlín se estima que alrededor de 80.000 personas están involucradas en el tema de agricultura urbana, en Boston unos 10.000 y en Nueva York hay un millar de huertos comunitarios en terrenos públicos [5]. Hace una década se estimaba que unos 800 millones de personas practicaban $\mathrm{AU}$, de los cuales 200 millones producían para el mercado y 150 millones estaban a dedicación completa.

\section{b) Desarrollo económico.}

Se generan unos ingresos con la actividad agraria que, en ocasiones, son los únicos existentes $\mathrm{y}$ otras veces complementarios, para estratos de población marginada. La creación de puestos de trabajo debe encuadrarse dentro de las políticas contra el desempleo. En ambos casos son hechos a tener en cuenta en situaciones de crisis económicas. Las políticas financieras y fiscales tienen también un papel relevante en este apartado [6].

\section{c) Medio ambiente y paisajismo.}

La actividad de las plantas a través de la función clorofílica captando $\mathrm{CO}_{2}$, devolviendo oxígeno y fijando partículas de polvo y metales pesados, es de consideración dentro 
de las acciones en la lucha contra la contaminación. Los efectos de aislamiento térmico y acústico se complementan con el reciclado de la emisión de gases de calefacción para invernaderos en edificios. La mejora del paisaje incrustando el verde en las masas grises de hormigón y cristal, es otra faceta de interés [4].

\section{d) Salud.}

La AU ayuda a lograr una dieta nutritiva más equilibrada al incorporar hortalizas frescas. Al mismo tiempo, el ejercicio físico realizado es saludable y el paisaje verde se considera un factor positivo contra el estrés y que mejora de la salud mental. Los servicios sociales de distintos países utilizan la AU como terapia para rehabilitación de drogadictos y personas con desequilibrios mentales [7].

e) Planificación urbana y normativa.

La agricultura necesita un espacio físico para su desarrollo, por ello los planificadores de la ciudad deben tenerla en cuenta buscando un equilibrio con la zona edificada, inclusive dentro de la misma.

Las normas sobre uso de espacio urbano, deben recoger explícitamente su utilización, acotando y reservando las adecuadas proporciones para actividades agrarias, evitando con ello conflictos de ilegalidad y alegalidad.

\section{f) Ocio y recreo.}

La naturación urbana implica incorporar la naturaleza a nuestro entorno inmediato y este viene siendo un deseo cada vez mayor entre los que viven alejados del campo. Los espacios verdes y abiertos permiten esa relajación y al mismo tiempo ocupan parte de nuestra vida ociosa y sedentaria.

Centros hospitalarios y comunidades vecinales impulsan la incorporación de la agricultura en su ambiente próximo.

\section{g) Relaciones sociales.}

Nuestro mundo de comunicación y redes sociales fomenta, paradójicamente, el individualismo y las escasas relaciones con nuestros vecinos geográficos. Se necesitan actividades conjuntas, políticamente neutras, que fomenten esa socialización y comunicación, con espíritu comunitario. El huerto urbano tiene también un elemento de relaciones inter-generacionales donde abuelos, padres e hijos comparten actividades conjuntas.

\section{h) Educación y formación.}

La actividad agraria es una escuela de formación permanente sobre la naturaleza. Cada vez son más frecuentes los planes de formación en los que se incluye, y los colegios que tienen huertos urbanos y clases prácticas que muestran los conocimientos básicos agrarios [8].

\section{CASOS CON GOBERNANZAS MULTIFACÉTICAS}

Con una visión holística de la gobernanza podemos considerar dos grandes áreas, una en sentido horizontal y otra vertical. La gobernanza horizontal se enfoca hacia las interacciones políticas y acciones entre actores que se encuentran en un determinado plano de gestión. Entendemos con ello a nivel de barrio, distrito, municipio, provincia, región, país o supranacional.

La gobernanza vertical se refiere a la interacción entre actividades desarrolladas entre los distintos niveles.

Consideramos a título de ejemplo el nivel de coordinación o conflictos entre las políticas locales municipales, las provinciales o nacionales.

En ambos flujos de gobernanzas, se identifica un núcleo o germen dinamizador de las políticas complementarias que progresivamente se va expandiendo, apoyándose en sinergias.

En los países en vías de desarrollo, el caso de la Agricultura Urbana muestra una variedad de acciones que pueden encuadrarse en el modelo de gobernanzas [9]. Veamos algunos ejemplos actuales, que pueden considerarse paradigmáticos. En Bolivia, el municipio de El Alto, ha triplicado su población en las tres últimas décadas, llegando a los 900 mil habitantes. La falta de infraestructuras y el fuerte crecimiento ha generado una población marginal, con problemas de abastecimiento alimentario, sanitarios y desempleo. Hay algunas iniciativas, como el proyecto de huertos familiares para la producción de hortalizas, financiado por Bélgica, durante el periodo 2004-2008 [9].

La dinámica de gobernanza vertical, está llevando a involucrar otros niveles de la Administración. En 2009 se incorporaba a la Constitución Boliviana el Derecho a la Alimentación y en 2014 ponía en marcha un Programa Nacional de Agricultura Urbana. Municipios como La Paz, Santa Cruz y Oruro están siguiendo los pasos comentados anteriormente.

El plan de gobernanza horizontal, trata de lograr sinergias entre actores e instituciones locales: Universidades, Administración, Organizaciones comunitarias, entidades financieras, centros de rehabilitación social y empresas. Otro caso de interés es la Agricultura Urbana en la ciudad de Rosario (Argentina). La evolución ha sido lo opuesto al caso de El Alto en Bolivia. Al igual que en otras grandes metrópolis, las crisis económicas han producido un empobrecimiento de sus habitantes, que disfrutaban de un elevado nivel de bienestar. El tejido industrial (químico, papel, acero) fue desapareciendo, el nivel de desempleo fue aumentando y la población empezó a emigrar, y de la población que permanecía, más de la mitad estaba por debajo del umbral de la pobreza. Como primera respuesta, el Municipio, en colaboración con el INTA y la ONG local CEPAR, se apoyaron en el programa Pro-Huerta y lanzaron un proyecto más amplio de Agricultura Urbana. La gobernanza horizontal consiguió aunar las fuerzas locales y en dos años existían 800 huertos comunitarios que abastecían de hortalizas a 40.000 personas (FAO 2014).

El modelo de gobernanza se ha ido ampliando, incorporando instituciones públicas y privadas, tanto de ámbito nacional (Universidad, Fundaciones) como internacionales (Fundación RUAF), habiendo obtenido el

Premio de Buenas Prácticas del Programa de la ONU para asentamientos urbanos. Su sistema operativo está siendo replicado en otras ciudades argentinas, como Buenos Aires y por las autoridades nacionales en programas de apoyo a la agricultura urbana.

La Habana se considera la ciudad más verde de Latinoamérica [9], y la opción de la Agricultura

Urbana vino provocada por causas externas que afectaron a todo el país. La caída del muro de Berlín y el colapso de los países de economía central planificada, deterioraron las relaciones económicas de Cuba con ese bloque, iniciándose lo que se denomina "periodo especial". A ello se unió el bloqueo comercial 
de EEUU, llevando a una gran escasez de alimentos y su racionamiento.

La agricultura tradicional productiva basada en el empleo de combustibles, fertilizantes y agroquímicos, sería obligada a reciclarse, utilizando sus propios recursos naturales. La ciudad de La Habana sufrió un desabastecimiento severo, y entre las soluciones más factibles se encontró la agricultura urbana, mediante los denominados cultivos Organopónicos en contra posición a los hidropónicos que sí utilizan fertilizantes y agroquímicos en las soluciones acuíferas.

De esta forma, lo que surgió como imperativo de supervivencia se ha convertido en un programa, que en 2013 proporciono 6.700 toneladas de alimentos, distribuidos en escuelas, hospitales y otras instituciones [9].

La gobernanza a nivel nacional corresponde al Ministerio de Agricultura, en el que se integra el Grupo Nacional de Agricultura Urbana y Suburbana, donde participan además otros seis ministerios y 16 instituciones científicas con 53 subprogramas sobre el tema.

El reto de la gobernanza, en este caso, es el pesado aparato burocrático que puede limitar la respuesta a las necesidades de la población. Algunas de las unidades productivas en La Habana consideraban que los objetivos marcados no eran compatibles con los medios y apoyos recibidos. En este punto, la coordinación de las gobernanzas entre el sector público y el privado será clave para la sostenibilidad del sistema de agricultura urbana.

En estos momentos la FAO estima que unos 90 mil vecinos están involucrados en agricultura urbana, bien en cultivos o en cría de animales domésticos. La venta de productos frescos tiene un radio comercial de 5 kilómetros, predominando las ventas directas productor-consumidor, con ferias y mercados estatales, así como a instituciones públicas (colegios, hospitales).

Se abre una nueva ventana, donde la oferta en calidad y cantidad puede permitir acceder al sector de hostelería y a los nuevos mercados. Los nuevos canales incluyen la venta ambulante (carretilleros), donde se alcanzan mayores precios y por consiguiente mayor rentabilidad para los productores, con un mejor servicio a los consumidores.

Todo ello exige un nuevo planteamiento de la gobernanza actual que debe resolver si se mantienen los modelos tradicionales o se complementan con las nuevas oportunidades. Quién y cómo se harán los reajustes, es uno de los interrogantes.

La ciudad de México es el centro de convergencia de distintos tipos de gobernanza. Como núcleo urbano, el DF tiene una población próxima a los 10 millones de habitantes, que se amplía a más de 20 con el área metropolitana. Simultáneamente, la capitalidad del país le confiere ser el punto radial para gobernanzas a nivel nacional.

Mención especial merece el capítulo "suelo de conservación" reserva creada en 1992 que abarca más de 300 km2

En la República Democrática del Congo, la prioridad ha sido asesorar a los Comités Municipales en el proceso de regularización de los títulos de propiedad de tierras para horticultura integrada en las actividades de agricultura, bosques y humedales, y se encuentra bajo la presión de invasiones continuas para cultivos y asentamientos furtivos.

A nivel nacional, la Secretaria de Desarrollo Rural y Equidad para las Comunidades (SEDEREC) fomenta el desarrollo agropecuario, agroindustrial y comercial en el medio rural. A nivel local de la Ciudad de México y con el problema ambiental subyacente, hay una prioridad hacia la agricultura ecológica, con un Programa de Ordenamiento Ecológico y una Ley Ambiental.
Se trata de conjugar las gobernanzas del Municipio y el Gobierno Federal en favor de la agricultura, la salud y el medio ambiente. Existe un programa de certificación ecológica (denominado "sello verde") aplicado a la agricultura urbana.

En Ecuador, Quito fue una metrópoli pionera, y en el año 2000 se produjo la "Declaración de Quito", en favor del desarrollo de la agricultura urbana, promovida por la reunión de nueve países Latinoamericanos [9]. En el año 2000 el proyecto piloto "El panecillo" fue estimulado por el Ayuntamiento de Quito, ubicando la agricultura en terrenos marginales.

Posteriormente la Agencia Metropolitana de Promoción Económica (CONQUITO), estimula una serie de proyectos como AGRUPAR, que actúa en los diferentes distritos de la ciudad. Los agricultores proceden de los movimientos migratorios y personas desempleadas, recibiendo cursos de capacitación y ayudas para semillas. Predomina la venta directa y se organizan las denominadas"bioferias" para la venta de los excedentes de producción.

No obstante, hay un punto débil en la coordinación de las gobernanzas. El Ministerio de Agricultura no incluye actividades urbanas en sus programas y el municipio de Quito no las contempla en su planificación urbana. Queda por tanto en una situación "alegal", donde se tolera pero no ofrece garantías necesarias para un mayor desarrollo.

La FAO ha venido asesorando en la gestión de gobernanza a municipios de ciudades reconocidas. En el Cairo, el programa de "Alimentos verdes en azoteas verdes", apoya el cultivo de hortalizas, sustentadas en la cascara de arroz, arena y musgo de turba. Simultáneamente se constató una diferencia de temperatura de $7^{\circ} \mathrm{C}$ en el aislamiento de las azoteas verdes en relación a las otras.

En Kigali (Ruanda) se asesoró a los servicios municipales en el diseño del plan urbanístico que contemplaba el desarrollo urbano con las actividades agrarias.

En la República Democrática del Congo, la prioridad ha sido asesorar a los Comités Municipales en el proceso de regularización de los títulos de propiedad de tierras para horticultura integrada en las actividades agrarias. Otra faceta de apoyo a la agricultura urbana son los Servicios de Extensión Agraria, como el caso de Hanoi (Vietnam), con más de un centenar de agentes trabajando en ese campo, e incrementando la eficiencia de sus agricultores.

En Gaza y Cisjordania, los esfuerzos se vienen centrando en el apoyo a unidades de tratamiento de bajo coste que permitan regar huertos y jardines con aguas grises de cocinas.

En los Países Desarrollados, la situación de la Agricultura Urbana ofrece también un espectro multifacético, en cuanto a sus gobernanzas. Hay ciudades donde las autoridades e instituciones municipales y nacionales han sido promotoras de la Agricultura Urbana por diversas motivaciones.

Con el fin de garantizar el abastecimiento de la población, en una economía de guerra, países como EEUU y Reino Unido, entre otros, hicieron grandes campañas de promoción de Huertos Urbanos en las Primera y Segunda Guerra Mundial. Además de mentalizar a la población, se daban cursos de capacitación y se facilitaban factores productivos.

La crisis económica actual está llevando a grandes centros urbanos a retomar la agricultura urbana. Detroit ha sido un paradigma de empobrecimiento de la población por deslocalización de la industria automovilística.

Los municipios están respondiendo con regulaciones y estímulos que facilitan dichas iniciativas, incluso involucrándose directamente en la naturación de edificios oficiales. Además de 
incentivos fiscales (subvenciones, reducción de impuestos), pueden darse otras medidas como la simplificación de trámites burocráticos en la concesión de licencias.

En Francia, hay un interés creciente en la coordinación de políticas y programas desarrollados con la AU [10]. En España, al igual que en otros países del entorno, la Agricultura Urbana, viene siendo una práctica habitual desde hace siglos. Sin embargo, el desarrollo urbanístico ha ido arrinconando su actividad quedando reducida a una serie de guetos en las grandes urbes.

Dicha marginación es también evidente en el mundo académico, en los estudios y publicaciones, si la comparamos con la agricultura rural, objeto sistemático de análisis y debates en el área política y socioeconómica. Una reciente publicación en español [11] recogía 47 monografías de divulgación, 20 trabajos de recursos didácticos, 7 trabajos académicos, 60 artículos de revista y capítulos de libro y 13 enlaces web, incluyendo algunas internacionales. De forma más exhaustiva, ya en el 2001 algunas publicaciones recogían 65 casos de análisis como fuente de información básica [12].

En el área de gobernanza sobre Agricultura Urbana la iniciativa viene siendo de movimientos de la sociedad civil que, por diversos motivos (sociales, políticos, culturales o económicos), vienen consolidando grupos de huertos urbanos en el suelo. La Agricultura Urbana en altura (paredes, terrazas) es testimonial, aunque no debemos olvidar la gran tradición ornamental en los patios andaluces y cultivos en macetas $\mathrm{y}$ balcones.

Sin embargo, hay una carencia en el desarrollo de gobernanzas administrativas, donde los municipios e instituciones regionales y nacionales presten atención a una realidad. Deben establecerse ordenanzas y regulaciones que contemplen espacios en los planes urbanísticos y de ordenación territorial, donde la agricultura urbana tenga un reconocimiento legal y supere las barreras que ahora tiene que enfrentar.

\section{CONCLUSIONES}

La AU se encuentra en una etapa de despegue. Es un fenómeno de moda en la sociedad urbana y aparece en los medios de comunicación como actividad con un futuro prometedor.

La buena gobernanza urbana es un campo importante para su análisis. La nueva AU necesita de procesos innovadores para adaptarse al nuevo entorno. Los aspectos técnicos, de gestión, regulaciones urbanísticas, buenas prácticas agrarias y comerciales, necesitan aportaciones de expertos pluridisciplinares. El nuevo espíritu empresarial puede dinamizar el tejido social urbano, en ocasiones frustrado con los parámetros actuales de convivencia.

El reto es cómo lograr una gobernanza eficiente que pueda sortear los obstáculos más significativos, sin ocasionar problemas adicionales, ni recelos con el mundo rural. No deben contemplarse las actividades agrarias urbana y rural como desarrollos alternativos, aunque hay planteamientos que abogan por la soberanía alimentaria de las urbes, en determinados productos.

\section{REFERENCIAS}

[1] DICKSON DESPOMMIER “Vertical Farm” Martín Press, 2010.

[2] RAE Diccionario de la Real Academia de la Lengua Española. 2014.
[3] COLOMER, J.M., NEGRETTO, G.L. "Gobernanza con poderes divididos en America Latina" Revista Centro de Investigación y Docencia Económicas. (2003)

[4] GUY PETERS, B. "La capacidad para gobernar ¿retrocediendo hacia el centro?" Revista CLAD. Reforma y Democracia. n²7 octubre. 2003.

[5] DUCHEMIN, E., WEGMUlLER, F., LEGAULT, M. "Urban Agriculture: multidimensional tools for social developments in poor neighbourhoods" http: factsreports.revues.org governance. 2008.

[6] GAGO, A., Y LAVANDEIRA, X. "La reforma fiscal verde" Mundiprensa. 1999.

[7] KOSHIMIZU, H "Urban green and human health" in Briz J, Koehler M, De Felipe I Green Cities in the World. Ed. Agrícola pp. 163-172. 2014.

[8] HENDRICKSON, M. K., PORTH, M. "Urban Agriculture: Best practices and possibilities" University of Missouri Extension. 2012.

[9] FAO, "Ciudades verdes en América Latina y el Caribe"(2014).

[10] MS Rojo, AZ Moratalla, NM Alonso, $\underline{\mathrm{VH} \text { Jimenez. }}$ Pathways towards the integration of periurban agrarian ecosystems into the spatial planning system. Springer. December 2014.

[11] RECIDA “Guía de recursos: agricultura urbana, huertos urbanos, huertos escolares" (2014).

[12] SMIT J., NASR J., RATTA A. "Urban Agriculture: Food, Jobs and Sustainable Cities". The Urban Agriculture Network Inc (UNDP). (2001) World Green Infrastructure. 